\title{
Análisis talla-estructurado de los cambios de abundancia en Merluccius gayi gayi entre 1992 y 2000
}

\author{
Claudio Gatica \& Luis Cubillos \\ Instituto de Investigación Pesquera. Casilla 350, Talcahuano, Chile \\ e-mail: cgatica@inpesca.cl
}

RESUMEN. Se analiza la condición actual del stock de merluza común (Merluccius gayi gayi) entre 1992 y 2000 , utilizando un modelo de evaluación talla-estructurado, evaluando las ventajas y desventajas del enfoque de modelación utilizado, que consiste en un modelo general de dinámica poblacional por edad y sexo, modelos para las observaciones de capturas anuales, composición por tamaños de la pesquería industrial de arrastre y composición por tamaños de los lances en los cruceros de evaluación acústica. El proceso de estimación permite la determinación simultánea de los parámetros mediante funciones de log-verosimilitud, penalizaciones, restricciones e información a priori de algunos parámetros. Los indicadores de la situación del stock muestran que la población está en un período estable de alta abundancia y biomasa, con una tendencia creciente en la abundancia de la fracción adulta de 1993 a 1998. Esta situación es producto de una serie de reclutamientos altos entre 1993 y 1997. En 1998 y 1999 el reclutamiento fue menos abundante, fluctuando entre 358 y 430 millones de individuos, mientras que en el 2000 hay un incremento de 816 millones de reclutas. Esta serie de reclutamientos en conjunto con tasas de explotación moderadas a bajas, habría posibilitado el incremento de la biomasa total de la merluza durante los años noventa. Con estos antecedentes, es oportuno saber cómo se puede mejorar el conocimiento del recurso y su dinámica poblacional, descubriendo fuentes de incertidumbres y variabilidad para la abundancia y examinar procesos que deben incorporarse en la herramienta de valoración. Este modelo se puede considerar como una herramienta alternativa que además de cuantificar la abundancia y biomasa entre otros indicadores, permitiría trabajar en la hipótesis de dinámica poblacional.

Palabras claves: merluza común, evaluación de stock, composición por talla, Chile.

\section{Size-structured analysis of abundance changes in Merluccius gayi gayi between 1992 and 2000}

\begin{abstract}
The present situation of the Chilean hake (Merluccius gayi gayi) is analyzed for the period between 1992 and 2000 through a length-based stock assessment model. The advantages and disadvantages of the model are analyzed, which consist of a general model of population dynamics structured by age and sex, models for the observations of total annual catches, fishery and survey length composition, and abundance from hydroacoustic surveys. The estimation processs allow a simultaneouly estimation of the parameters through log-likelihood functions, penalties, constraints, and a priori information for some parameters. The stock indicators show a population in a period of high abundance and biomass, after a growing trend in the adult abundance of the stock from 1993 to 1998 . This situation has been a consequence of higher recruitments occurring between 1993 and 1997. In 1998 and 1999 the recruitment was less abundant, ranging from 358 to 430 millions of individuals, while in 2000 it increases until 816 millions of recruits. This series of good recruitments in addition with moderates and lower exploitation rates would have generated the favorable increment in the total biomass of Chilean hake during the 1990s. Under this scenario, is opportune to know how we can improve the knowledge of the resource and their populational dynamics, by detecting sources of uncertainties and variability for the abundance and by examining processes that should be incorporated in the assessment tool. This model can be seen as an alternative tool that besides quantifying the abundance and biomass among other indicators, would allow to work on hypothesis of populational dynamics.
\end{abstract}

Key words: common hake, stock assessment, lenght composition, Chile. 


\section{INTRODUCCIÓN}

La merluza común (Merluccius gayi gayi) es el principal recurso demersal en la zona centro-sur de Chile y es explotado tanto por el sector industrial como el artesanal. La pesquería de este recurso se encuentra declarada en plena explotación y ha sido administrada mediante cuotas globales anuales de captura, y a contar del 2001 bajo el régimen de límites máximos de captura por armador en el caso de la pesquería industrial. La fijación de cuotas se basa en criterios biológicamente aceptables estimados a partir de una evaluación cuantitativa de la abundancia del stock (Payá et al., 1998).

La principal herramienta de evaluación indirecta utilizada para estimar la abundancia de merluza común ha sido el análisis secuencial de poblaciones (ASP). A partir de 1991, se incorporó al análisis, información auxiliar para estabilizar y calibrar los resultados del ASP, utilizando en los años 1992 y 1993 la captura por unidad de esfuerzo (CPUE) de la flota de barcos de más de 1200 HP (Payá et al., 1992; Cubillos et al., 1994). La CPUE constituye un índice de abundancia relativa que se relaciona teóricamente con la biomasa media explotable del stock, de acuerdo al coeficiente de capturabilidad $q$. Sin embargo, existen importantes limitaciones con el uso de la CPUE, debido a cambios probables en $q$ (Hilborn \& Walters, 1992; Fréon \& Misund, 1999), optándose a contar de 1995 por el uso de estimados directos de la abundancia por grupos de edad, a partir de las evaluaciones de biomasa realizadas mediante hidroacústica (Payá et al., 1998). A su vez, la incertidumbre en los datos de entrada comenzó a ser incorporada en la evaluación, en lo que se ha denominado ADASIM (Payá et al., 1998).

Aunque el ASP ha sido la técnica más utilizada $\mathrm{y}$ frecuentemente aplicada para evaluar stocks (Megrey, 1989), uno de los principales problemas de este método subyace en el supuesto de que las capturas en número por grupos de edad son cantidades observadas sin error. Aunque ADASIM es un esquema ADAPT de estimación, y puede incorporar la incertidumbre relacionada con errores de observación, la evaluación se realiza en forma independiente para machos y hembras dada las características diferenciales en las tasas de crecimiento, mortalidad natural y probablemente en el patrón de explotación. En consecuencia, hasta el momento no se ha intentado un análisis integrado que permita la evaluación simultánea del stock de machos y hem- bras, ni se han evaluado las consecuencias de este enfoque integrado en las estimaciones poblacionales totales.

Una alternativa para la evaluación es la utilización de los denominados modelos estadísticos de captura a la edad, donde se supone que todas las cantidades observadas son medidas con error de observación y que el modelo de los procesos se puede relacionar con dichas cantidades a través de modelos para las observaciones (Fournier \& Archibald, 1982; Methot, 1986; Kimura, 1990; Quinn \& Deriso, 1999). Las ventajas se asocian principalmente con la posibilidad de una estimación estadística simultánea de la abundancia inicial de todas las cohortes y de las tasas de mortalidad por pesca anuales, entre otros parámetros, por medio de la minimización de una función objetivo basada en criterios estadísticos, como los estimadores de máxima verosimilitud (Kimura, 1990). Además, se puede estimar la varianza y por lo tanto intervalos de confianza para todos los parámetros estimados, que son condicionales a los datos de captura a la edad y a la estructura del modelo (Quinn \& Deriso, 1999).

No obstante lo anterior, los datos de captura a la edad aún pueden contener errores de observación relacionados con las determinaciones de la edad, y con la transformación de los datos de composición por longitudes en composición por edad utilizando claves talla-edad. En este trabajo se implementa un modelo de evaluación edad-estructurado para la dinámica de la población, que utiliza datos observados de la composición por longitudes de las capturas y de la población para estimar la abundancia de las clases anuales y las tasas de mortalidad por pesca. En consecuencia, se puede decir que los cambios de abundancia que emergen del modelo de estimación son básicamente talla-estructurados ya que las estimaciones se basan en el ajuste de la información de composición por tamaños de la población, sin la necesidad de conocer previamente la composición por edad de las capturas. Bajo este enfoque, es posible la estimación de una estructura de tamaños que es comparable estadísticamente con la estructura de tamaños observada en la pesquería y con la de la población, obtenida a partir de los lances de evaluación de los cruceros acústicos y que representan posteriormente, la composición de tamaños del estimado acústico (Lillo et al., 2001).

El modelo de dinámica presenta cuatro componentes básicos: a) un modelo de dinámica poblacional estructurado por edad y sexo, que a tra- 
vés de modelos para las observaciones permite generar estimaciones de capturas anuales, composición por tallas en las capturas, abundancia total y composición por talla en los cruceros de evaluación acústica, b) funciones de log-verosimilitud para la estructura del error en los datos observados, c) penalizaciones, restricciones e información a priori para algunos parámetros, y d) un proceso de estimación, a través de la minimización de una función objetivo general. El modelo considera la existencia de una sola unidad de stock, con diferencias por sexo para el crecimiento, las tasas de mortalidad natural y la selectividad al arte de pesca.

Los objetivos de este trabajo son analizar las ventajas y desventajas del modelo y enfoque de evaluación aquí utilizados para evaluar el stock, en relación con la comprensión de los cambios de abundancia del stock de merluza común.

\section{MATERIALES Y MÉTODOS}

\section{Área de estudio}

El área de estudio comprende la unidad de pesquería del recurso merluza común, definida entre los $29^{\circ} 10^{\prime} \mathrm{S}$ y $\operatorname{los} 41^{\circ} 28,6^{\prime} \mathrm{S}$ (Fig. 1), donde se considera que tanto la flota industrial como artesanal operan sobre una sola unidad de stock.

\section{Capturas anuales y composición por tamaños}

La información utilizada se obtuvo de diversas fuentes: proyectos financiados por el Fondo de Investigación Pesquera (FIP) y la base de datos del Sistema de Información de Merluza Común (SIMEC) del Instituto de Investigación Pesquera. Se utiliza las capturas totales anuales (artesanal más industrial) del periodo 1992-2000 y datos de la composición por tamaño anuales, que representan la estructura de tallas de las capturas obtenidas por la pesquería industrial de arrastre. Esta información, proviene tanto de datos operacionales por lance registrados en bitácoras de pesca y muestras de frecuencias de tamaños obtenidas en cada lance, a bordo de las embarcaciones arrastreras.

Los datos de frecuencias de tallas se obtienen en machos y hembras, clasificando la longitud total de los peces en intervalos de $1 \mathrm{~cm}$. La información colectada durante el período de estudio cubre en promedio un $10 \%$ del desembarque industrial. Las embarcaciones a muestrear son asignadas aleatoriamente, según la disponibilidad de tripula- ción y habitabilidad que presentaran al momento de cada embarque. Por problemas de disponibilidad no se cuenta con registros e información para obtener la composición por tamaños de las capturas del sector artesanal. Sin embargo, esta información ingresa al modelo de evaluación de stock en el dato de captura total (sector artesanal e industrial).

\section{Estimaciones de abundancia por acústica}

Se utiliza las estimaciones de abundancia realizadas en los años 1993, 1995, 1997, 1999 y 2000; y la composición por tamaño obtenidas a partir de los lances de pesca orientados tanto para la identificación de los eco-registros acústicos como para determinar la estructura de tamaños de la biomasa cuantificada (Lillo et al., 1993, 1998, 2000; Payá et al., 1995).

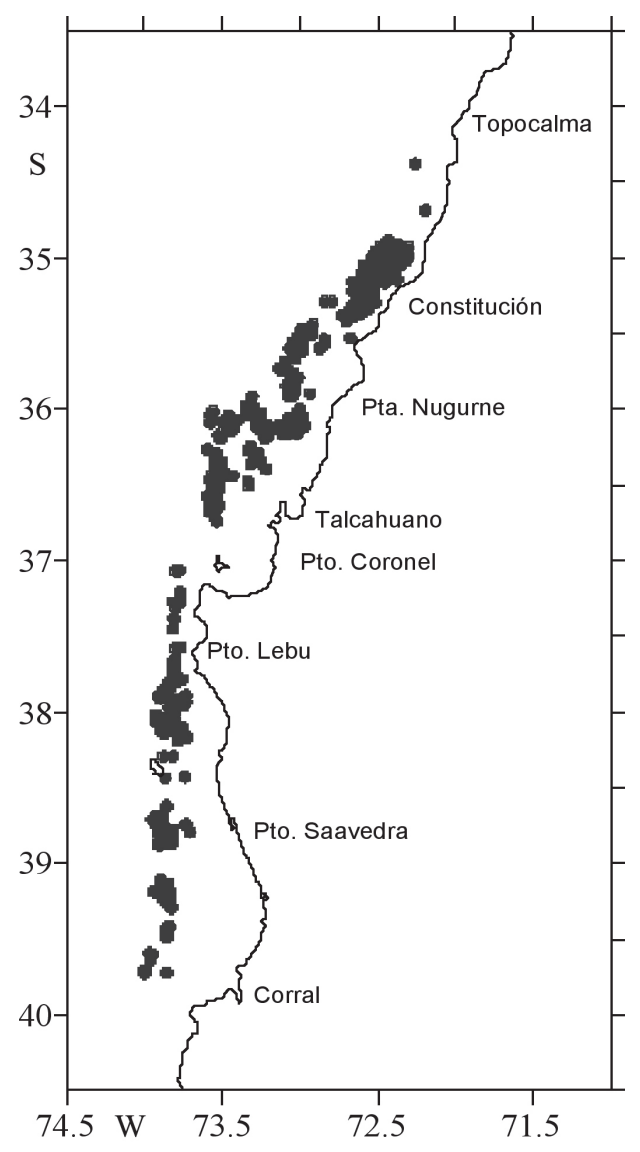

Figura 1. Principal área de operación de la flota merlucera.

Figura 1. Principal area of operation of the fleet of common hake. 


\section{Modelo de evaluación de stock}

El modelo es edad-estructurado y utiliza la ecuación de captura de Baranov como modelo operacional (Deriso et al., 1985; Hilborn \& Walters, 1992; Schnute \& Richards, 1995). El modelo supone la existencia de una sola pesquería y considera las diferencias sexuales en la tasa de mortalidad natural, crecimiento y vulnerabilidad al arte de pesca. El modelo es implementado considerando que la dinámica del stock es edad-estructurada y que el crecimiento es un atributo de la población de tal forma que es posible utilizar la información de composición por tallas para modelar la dinámica poblacional del stock de merluza común.

\section{Dinámica poblacional}

\section{Abundancia}

Se estima que la abundancia de machos y hembras disminuye exponencialmente de acuerdo con el siguiente modelo:

$$
\begin{gathered}
N_{i+1, j+1, s}=N_{i, j, s} \exp \left(-Z_{i, j, s}\right) \\
1 \leq i \leq I ; 1 \leq j \leq J \\
N_{i+1, J, s}=N_{i, J-1, s} \exp \left(-Z_{i, J-1, s}\right)+N_{i, J, s} \exp \left(-Z_{i, J, s}\right)
\end{gathered}
$$

$$
1 \leq i \leq I ; j=J
$$

donde: $N_{i, j, s}$ representa la abundancia en número de individuos de edad $j(\mathrm{j}=1,2, . ., J)$ en el stock de sexo $s$ (machos: $s=m$; hembras: $s=h$ ) a comienzos del año $i(i=1,2, \ldots, I)$. La abundancia del primer grupo de edad de los machos en todos los años, se estima por:

$$
N_{i, 1, s=m}=\pi_{\mathrm{i}} * N_{i, 1}
$$

y el de hembras por:

$$
N_{i, 1, s=h}=\left(1-\pi_{\mathrm{i}}\right) * N_{i, 1}
$$

En ambos casos $\pi_{\mathrm{i}}$ representa la proporción de machos del primer grupo de edad, y su complemento a la proporción de hembras. Un modelo similar fue utilizado para la abundancia a la edad en el primer año. En este contexto, aunque la abundancia inicial de machos y hembras podría haber sido estimada sin necesidad de recurrir a la proporción sexual, para luego obtener la abundancia total (suma de las partes), se adopta un esquema de estimación que divide el total entre las partes. Luego, la abundancia de las partes (machos y hembras) queda determinada por la mortalidad total de cada sexo (Ecuaciones 1 y 2). La contribución a la log-verosimilitud en el proceso de estimación de la proporción sexual viene dada por la siguiente expresión:

$$
\ell_{1}=\lambda_{1} \sum_{i}\left(\pi_{i}-0,5\right)^{2}
$$

donde: $\lambda_{1}$ representa una variable relacionada con la varianza $\left(\lambda=1 / 2 \sigma^{2}\right)$ y se introduce como fuente no-informativa de esta información en la función de log-verosimilitud total. El valor 0,5 implica que en promedio, la proporción sexual es 1:1 a la edad de reclutamiento.

\section{Mortalidad}

La tasa de mortalidad total $\left(Z_{i, j, s}\right)$, es modelada de la siguiente manera:

$$
Z_{i, j, s}=F_{i, j, s}+M_{s}
$$

donde: $F_{i, j, s}$ es la tasa de mortalidad por pesca y $M_{s}$ es la tasa de mortalidad natural. Esta última se considera conocida en cada sexo, y constante entre años y grupos de edad, correspondiendo en el caso de los machos $M_{s=m}=0,43$ año ${ }^{-1}$ y en hembras $M_{s=h}=0,26$ año-1 (Payá et al., 1992, 1993).

En el caso de la mortalidad por pesca se utiliza un modelo multiplicativo compuesto por un factor edad-específico $\left(v_{j, s}\right)$ y otro año específico $\left(F_{i, s}\right)$ que permite reducir el número de parámetros a estimar. Esta hipótesis, llamada de separabilidad (Doubleday, 1976; Deriso et al., 1985), lleva a la siguiente expresión:

$$
F_{i, j, s}=v_{j, s} * F_{i, s}
$$

y permite reducir el número de parámetros desconocidos cuando $v_{j}$ es constante entre años. Los valores edad-específicos se estimaron libremente para $g$ grupos de edad, sin suponer un modelo particular a priori (e.g. logístico, gamma, u de otro tipo). La modelación de los patrones de explotación edadespecífico es realizada libremente según la metodología descrita por Ianelli \& Zimmerman (1998), estimándose los valores de $v_{j}$ para $g$ grupos de edad $(j$ $\leq g$ ), y considerando que los grupos de edad más 
viejos $(j>g)$ tienen igual valor que a la edad $g$. Los $g$ valores de $v_{j}$ fueron estimados en la escala logarítmica, de manera que:

$$
v_{j}=\exp \left(\eta_{j}\right)
$$

donde: $\eta_{j}=0$ para $\mathrm{j}>\mathrm{g}$, con la siguiente limitación

$$
\sum_{j} \eta_{j}=0
$$

La contribución a la log-verosimilitud consta de dos partes. La primera corresponde a una doble diferenciación para suavizar:

$$
\ell_{v, 1}=\lambda_{v, 1}^{x} \sum_{j}\left(\eta_{j+2}^{x}+\eta_{j}^{x}-2 \eta_{j+1}^{x}\right)^{2}
$$

y la segunda es una función que permite modificar el grado de declinación del patrón de explotación con la edad, i.e.

$$
\ell_{v, 2}=\lambda_{v, 2}^{x} \sum_{j}\left(\eta_{j}^{x}<\eta_{j-1}^{x}\right)\left(\exp \left(\eta_{j}^{x}\right)-\exp \left(\eta_{j+1}^{x}\right)\right)^{2}
$$

El superíndice $x$ denota un patrón de explotación particular (sexo, pesquería y crucero de evaluación). Las constantes $\lambda_{v, 1}$ y $\lambda_{v, 2}$ representan coeficientes de énfasis no informativos para el proceso de estimación.

Debido a que el patrón de explotación edad-específico $\left(v_{j}\right)$ puede afectar las estimaciones de abundancia y de mortalidad cuando se estiman constante entre años, se probó un modelo alternativo que permite que el patrón de explotación pueda ser variable en el tiempo, modelándose su variación mediante un proceso de caminata aleatoria :

$$
v_{i+k, j}=v_{i, j} * \exp \left(\gamma_{i, j}\right)
$$

donde: $k$ indica el número de años consecutivos con igual patrón de explotación edad-específico y

$$
\gamma_{i, j} \approx N\left(0, \sigma_{v}^{2}\right)
$$

En el proceso de estimación, la siguiente regularidad debe ser agregada:

$$
\ell_{v, 3}=\lambda_{v, 3}^{x} \sum_{i=1}^{T} \sum_{j}^{J}\left(\eta_{1+i \cdot k, j}^{x}\right)^{2}
$$

donde: $T$ representa el número de $k$ grupos de años consecutivos en que el patrón de explotación permanece constante, i.e. es el valor entero de la diferencia entre el último y primer año de la serie dividido por $k$. La contribución a la log-verosimilitud total de estos componentes para cada patrón de explotación viene dada por la suma de las Ecuaciones (11) a (12), i.e.

$$
\ell_{2}=\sum_{x}\left[\ell_{v, 1}+\ell_{v, 2}+\ell_{v, 3}\right]
$$

\section{Crecimiento en longitud y clave edad-talla}

La longitud media a la edad para el sexo s es descrita por el modelo de von Bertalanffy:

$$
L_{j, s}=L_{\infty, s}\left[1-\exp \left(-k_{s}\left(j-t_{0, s}\right)\right)\right]
$$

donde: $L_{\times}$es la longitud asintótica, $k$ es el coeficiente de crecimiento, y $t_{0}$ es la edad hipotética cuando la longitud es igual a cero. Por otra parte, la desviación estándar de la longitud a la edad $\left(\sigma_{j}\right)$ se estimó mediante la siguiente relación que incorpora el coeficiente de variación a la edad $C V_{j}$ que se estima como parámetro desconocido.

$$
\sigma_{j, s}=C V_{j, s} * L_{j, s}
$$

La siguiente función se incorporó en el proceso de estimación:

$$
\ell_{3}=\lambda_{C V} \sum_{j}\left(C V_{j}-0,06\right)^{2}
$$

En otras palabras, se limita a que el $C V$ tenga una media de 0,06 para dar mayor peso al ajuste de las observaciones de composición por tamaños. Es posible destacar que $C V$ promedio de 7 y $9 \%$ ha sido encontrado en estudios de edad y crecimiento (Ojeda et al., 1997).

La probabilidad que tiene un pez de edad $j$ de pertenecer a un intervalo de longitud $l$ fue modelada mediante una función de distribución normal de la longitud a la edad. Esta función permite definir para el rango de edades y tallas una matriz de probabilidad $\left(\psi_{j, l}\right)$ en machos y otra en hembras de la siguiente forma:

$$
\psi_{j, l}=\frac{1}{\sqrt{2 \pi * \sigma_{j}}} \exp \left[-\frac{1}{2 \sigma_{j}^{2}}\left(L_{l}-L_{j}\right)^{2}\right]
$$


donde: $L_{l}$ representa la marca de clase del intervalo de longitud $l$ y $L_{j}$ es la longitud media a la edad. Así, el número de ejemplares machos y hembras en el stock por clases de longitud $\left(N_{i, l}\right)$ puede ser estimada por:

$$
N_{i, l, s}=\sum_{j=1} \psi_{j, l, s} * N_{i, j, s}
$$

\section{Peso promedio a la edad}

El peso promedio a la edad se modeló utilizando la relación longitud-peso, donde $w$ es el peso promedio $(\mathrm{kg})$ a la longitud $L(\mathrm{~cm})$. Se utilizó $a_{m}=0,000197$ y $b_{m}=2,215$ en machos, y $a_{h}=0,000018$ y $b_{h}=2,764$ en hembras (SIMEC). Como el peso promedio es un estimado poblacional correspondiente a una media obtenida a partir de la información de un grupo de peces de una misma edad, se utilizó el siguiente modelo para estimar el peso promedio a la edad (Beyer, 1991), que incorpora el coeficiente de variación de la longitud a la edad:

$$
w_{j, s}=a_{s} * L_{j, s}^{b_{s}}\left[1+0,5 * b_{s}\left(b_{s}-1\right) C V_{j, s}^{2}\right]
$$

\section{Biomasa total, adulta y desovante}

La biomasa total $B_{i, s}^{t}$ para el sexo $s$ es:

$$
B_{i, s}^{t}=\sum_{j=1} N_{i, j, s} * w_{j, s}
$$

y la biomasa total del stock corresponde a la suma de las biomasas totales de machos y hembras. La biomasa adulta $B_{i, s}^{a}$ para el sexo $s$ queda definida a comienzos de cada año utilizando la siguiente expresión:

$$
B_{i, s}^{a}=\sum_{j=1} N_{i, j, s} * w_{j, s} \mu_{j, s}
$$

donde: $N_{i, j}$ es la abundancia en número, $w_{j}$ es el peso promedio a la edad, y $\mu$ es el vector de madurez correspondiente, informado por Cerna \& Oyarzún (1998). Ante la ausencia de estudios de madurez sexual en machos, se supone que éstos tienen una ojiva de madurez idéntica a las hembras.

La biomasa desovante $B_{i, s}^{d}$ queda definida como la biomasa adulta que sobrevive hasta el primero de agosto $(=7 / 12)$, periodo donde se produce la mayor actividad reproductiva del recurso (Payá et al., 1996):

$$
B_{i, s}^{d}=\sum_{j=1} N_{i, j, s} * w_{j, s} * \mu_{j, s} * \exp \left(-(7 / 12) Z_{i, j, s}\right)
$$

\section{Modelos de las observaciones}

\section{Captura y composición por tallas en la pesquería}

La captura en número por edad $\left(C_{i, j}\right)$ se modela mediante la ecuación de Baranov:

$$
\begin{gathered}
C_{i, j, s}=\frac{F_{i, j, s}}{Z_{i, j, s}}\left(1-\exp \left(-Z_{i, j, s}\right)\right) N_{i, j, s} \\
1 \leq i \leq I \text { y } 1 \leq j \leq J
\end{gathered}
$$

La estimación de composición por tallas $\left(p_{i, l}\right)$ de las capturas se estimó a partir de la captura por edad y la matriz de probabilidad edad-talla $\left(\psi_{j, l}\right)$ en machos y hembras:

$$
p_{i, l, s}=\sum_{j=1} \psi_{j, l, s} * p_{i, j, s}
$$

donde:

$$
\begin{gathered}
p_{i, j, s}=C_{i, j, s} / C_{i, s} \\
C_{i, s}=\sum_{j=1} C_{i, j, s}
\end{gathered}
$$

\section{Composición por tallas en los lances de los cruceros de evaluación acústica}

Las estimaciones de composición por tallas de los lances en los cruceros de evaluación acústica $\left(p_{i, l}^{H}\right)$, se generan a partir de la composición por edad estimada de la abundancia al momento del crucero $\left(p_{i, j, s}^{H}\right)$ y la matriz de probabilidad edad-talla $\left(\psi_{j, l, s}\right)$; esto es

$$
\begin{gathered}
p_{i, j, s}^{H}=\frac{q_{s}^{H} v_{s}^{H} N_{i, j, s} \exp \left(-(7 / 12) Z_{i, j, s}\right)}{\sum_{j} q_{s}^{H} v_{s}^{H} N_{i, j, s} \exp \left(-(7 / 12) Z_{i, j, s}\right)} \\
p_{i, l, s}^{H}=\sum_{j=1} \psi_{j, l, s} * p_{i, j, s}^{H}
\end{gathered}
$$

donde: $q^{H}$ representa el coeficiente de capturabilidad para el crucero, el cual se considera igual a 1 para no ser estimado y $v^{H}$ corresponde al patrón de ex- 
plotación asociado al crucero de evaluación. El denominador de la Ecuación (27) representa la abundancia acústica $N_{i, j, s}^{H}$ de cada sexo.

\section{Funciones de log-verosimilitud}

Los parámetros desconocidos del modelo pueden ser estimados empleando funciones conocidas para la estructura del error de los datos observados, utilizando métodos de estimación de máxima verosimilitud. Para ello, es necesario describir cada componente de verosimilitud, suponiendo independencia en las observaciones.

La captura en peso (rendimiento, $Y_{i}$ ) se estimó multiplicando la captura en número a la edad por el peso promedio a la edad, i.e.

$$
Y_{i}=\sum_{j=1} w_{j}^{*} C_{i, j}
$$

Se considera que las capturas en peso siguen una función de densidad de probabilidad log-normal, y la función de log-verosimilitud negativa viene dada por:

$$
\ell_{4}=\lambda_{Y} \sum_{i=1}\left(\ln \left(Y_{i}^{\prime}\right)-\ln \left(Y_{i}\right)\right)^{2}
$$

donde: $Y^{\prime}{ }_{i}$ es la captura total en peso observada, en tanto $\lambda_{Y}=1 / 2 \sigma_{Y}^{2}$, y se considera determinado $a$ priori como un supuesto no informativo de la contribución de los datos de captura en peso a la log-verosimilitud.

La composición por tallas consiste en clasificar la muestra en categorías de tallas, por lo tanto la distribución del error adopta una función de densidad de probabilidad multinomial (Kimura, 1990; Fournier et al., 1990), i.e.

$$
\prod_{i, l}\left(p_{i, l}\right)^{n_{i, l}}
$$

donde: $n_{i, l}$ representa por definición, el número de individuos muestreados en el año $i$ que fueron de la clase de talla $l$. Para encontrar un estimador máximo verosímil de los parámetros del modelo, la cantidad a ser minimizada es la parte negativa de la función de log-verosimilitud (Kimura, 1990):

$$
\ell_{5}=-n_{i, l} \sum_{i=1}^{I} \sum_{i=1}^{n_{l}} p_{i, l}^{\prime} * \ln \left(p_{i, l} / p_{i, l}^{\prime}\right)
$$

donde: $p_{i, l}^{\prime}$ representa la composición por talla observada y $n_{i, l}$ corresponde al número de intervalos de clase. En la expresión, $n_{i, l}$ se reemplazó por $n$. Fournier \& Archibald (1982) y Methot (1986) sugieren un límite máximo ad hoc de $n=400$ debido a que en la práctica el tamaño de muestra para determinar la estructura de tallas puede ser mucho mayor. En el modelo se utilizó $n=200$, tanto para la composición por tamaño de las capturas como para la composición por tamaño de los lances de identificación en los cruceros de evaluación acústica. La log-verosimilitud de este tipo de información no es exactamente la Ecuación (32), sino más bien la suma de la función de log-verosimilitud de cada pieza de información por sexo y tipo de composición por tamaños (pesquería y crucero).

En el caso de la abundancia estimada por métodos acústicos, se adopta una función de densidad de probabilidad log-normal acústica $\left(N_{i, s}^{, H}\right)$, entonces la contribución de esta información a la log-verosimilitud viene dada por:

$$
\ell_{6}=\lambda^{H} \sum_{i}\left(\ln \left(N_{i, s}^{N_{i, s}^{H}}\right)-\ln \left(N_{i, s}^{H}\right)\right)^{2}
$$

donde: $\lambda_{H}$ es el "lambda" correspondiente al término de varianza de la abundancia acústica.

Se destaca que la abundancia inicial de cada una de las clases anuales modeladas se estimó por:

$$
N_{i, 1}=R * \exp \left(\varepsilon_{i}\right)
$$

que representa un proceso estocástico (geométrico), i.e.

La contribución de este error de proceso a la función de log-verosimilitud negativa viene dada por:

$$
\ell_{7}=\lambda_{R} \sum_{i=1} \varepsilon_{i}^{2}
$$

A su vez, el componente año-específico de la mortalidad por pesca por sexo fue modelado mediante un proceso estocástico alrededor de un valor promedio $(F)$ (geométrico), dado por:

$$
F_{i, s}=F_{s} \exp \left(\tau_{i, s}\right)
$$


donde: $\tau_{i, s}$ representa desviaciones interanuales (anomalías) de la media, considerando una distribución normal con media cero y varianza constante.

$$
\tau_{i, s} \approx N\left[0, \sigma_{, s F}^{2}\right]
$$

Por lo tanto, la contribución de este error de proceso a la log-verosimilitud es:

$$
\ell_{8}=\sum_{s}\left(\lambda_{F} \sum_{i} \tau_{i, s}^{2}\right)
$$

La función objetivo total a ser minimizada, viene dada por la suma de las funciones de log-verosimilitud negativa previamente descritas, i.e.

$$
\ell=\sum_{i} \ell_{i}
$$

\section{Análisis de sensibilidad}

Se realizó un análisis de sensibilidad que considera la comparación de seis modelos alternativos en relación a los parámetros de crecimiento de machos y hembras, y la construcción del modelo con patrón de selectividad constante y variable (Tabla 1), debido a que el ajuste se fundamenta en la información de composición por tamaños. La comparación de estos modelos se realizó sobre la base de los componentes de verosimilitud de la función objetivo y el Criterio de Información de Akaike (Hilborn \& Mangel, 1997). Los valores de "lambda" y su influencia en el ajuste del mejor modelo en términos de la desviación estándar se indican en la Tabla 2.

\section{Optimización no-lineal}

El algoritmo de estimación del modelo se implementó en AD Model Builder ${ }^{\odot}$ de la Otter Research Ltd (otter@island.net).AD Model Builder utiliza derivadas exactas respecto de los parámetros del modelo. Además, es muy útil para modelos con una gran cantidad de parámetros, provee estimaciones rápidas y precisas de la matriz Hessiana en el máximo (o mínimo), lo que permite contar con la matriz de covarianza, correlación y por lo tanto con límites de confianza de todos los parámetros.

\section{RESULTADOS}

El Criterio de Información de Akaike (AIC) indica que el modelo 1 es mejor en términos de ajuste y grado de explicación. Este modelo tiene como principales características el ser construido con un patrón de selectividad constante, que utiliza los parámetros de crecimiento de machos y hembras obtenidos desde el Sistema de Información de Merluza Común (SIMEC) del Instituto de Investigación Pesquera. En los modelos con patrón de selectividad variable (modelo 4, 5 y 6), el mejor modelo es el 4, esto se puede ver tanto por el valor AIC como por el componente de log-verosimilitud del modelo. Desde el punto de vista de los parámetros de crecimiento, se puede apreciar que las componentes de log-verosimilitud de la composición por talla para la pesquería y los cruceros de evaluación hidroacústica son los que presentan las mayores diferencias y en consecuencia afectan de forma más

Tabla 1. Parámetros de crecimiento utilizados para analizar la sensitividad de los modelos de machos (M) y hembras (H).

Table 1. Growth parameters used to analyze the sensitivity of the models for males $(\mathrm{M})$ and females $(\mathrm{H})$.

\begin{tabular}{|ccccccc|}
\hline Modelo & Sexo & Patrón selectividad & $\mathbf{L}_{\mathbf{x}}(\mathbf{c m})$ & $\mathbf{k}\left(\mathbf{a n ̃ o} \mathbf{-}^{-1}\right)$ & $\mathbf{t}_{\mathbf{0}}(\mathbf{a n ̃ o})$ & Fuente \\
\hline \multirow{2}{*}{1} & macho & Constante & 55,97 & 0,273 & $-0,06$ & SIMEC \\
& hembra & Constante & 64,3 & 0,206 & $-0,24$ & SIMEC \\
\hline \multirow{2}{*}{2} & macho & Constante & 57,4 & 0,288 & $-0,232$ & Aguayo \& Ojeda (1987) \\
& hembra & Constante & 78,1 & 0,153 & $-0,782$ & Aguayo \& Ojeda (1987) \\
\hline \multirow{2}{*}{3} & macho & Constante & 58,3 & 0,249 & $-0,749$ & Ojeda et al. $(1997)$ \\
& hembra & Constante & 81,89 & 0,119 & $-1,557$ & Ojeda et al. $(1997)$ \\
\hline \multirow{2}{*}{4} & macho & Variable & 55,97 & 0,273 & $-0,06$ & SIMEC \\
& hembra & Variable & 64,3 & 0,206 & $-0,24$ & SIMEC \\
\hline \multirow{2}{*}{5} & macho & Variable & 57,4 & 0,288 & $-0,232$ & Aguayo \& Ojeda (1987) \\
& hembra & Variable & 78,1 & 0,153 & $-0,782$ & Aguayo \& Ojeda (1987 \\
\hline \multirow{2}{*}{6} & macho & Variable & 58,3 & 0,249 & $-0,749$ & Ojeda et al. (1997) \\
& hembra & Variable & 81,89 & 0,119 & $-1,557$ & Ojeda et al. (1997) \\
\hline
\end{tabular}


significativa el término de la función objetivo (Tabla 3).

Los resultados corresponden al modelo más verosímil en relación a los parámetros de crecimiento y patrón de selectividad constante (Modelo 1). En términos generales, los seis modelos se comportaron de manera similar reflejando las mismas tendencias, variando sólo en algunos estimados.

El modelo predice adecuadamente la captura del período 1992-2000. La mayor diferencia entre el valor observado y esperado se produjo en 1993. En esta serie, las mayores capturas se presentaron en los dos últimos años, específicamente en 1999 y 2000 con 93 mil y 105 mil ton respectivamente (Fig. 2).

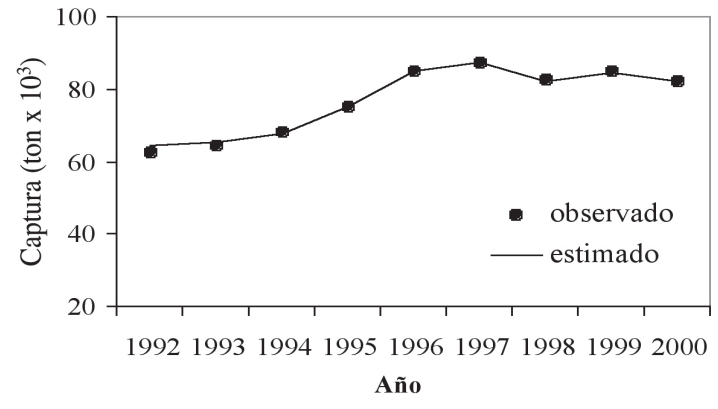

Figura 2. Capturas totales observadas y estimadas entre 1992 y 2000.

Figure 2. Total catch observed and estimated for 1992 -2000 period.

Tabla 2. Coeficientes de contribución a la verosimilitud (lambda) y su peso en el ajuste del modelo en términos de desviación estándar. Los valores de desviación estándar están en escala logarítmica y escala normal (asterisco).

Table 2. Contribution coefficients to the likelihood (lambda) and their weight in the model fit in terms of standard deviation. The values of standard deviation are in logarithmic scale and normal scale (asterisk).

\begin{tabular}{|clc|}
\hline Lambda & Descripción & Desviación estándar \\
\hline$\lambda_{1}$ & Término proporción reclutas entre machos y hembras & $0,02^{*}$ \\
$\lambda_{2}$ & Término de varianza del reclutamiento & 0,35 \\
$\lambda_{3}$ & Término de varianza de abundancia machos (crucero) & 0,04 \\
$\lambda_{4}$ & Término de varianza de abundancia hembras (crucero) & 0,04 \\
$\lambda_{5}$ & Término de varianza capturas (machos) & 0,01 \\
$\lambda_{6}$ & Término de varianza capturas (hembras) & 0,01 \\
$\lambda_{7} \mathrm{y} \lambda_{8}$ & Término de varianza curvatura y diferenciación Pesquería (machos) & 0,01 \\
$\lambda_{9} \mathrm{y} \lambda_{10}$ & Término de varianza curvatura y diferenciación Pesquería (hembras) & 0,01 \\
$\lambda_{11} \mathrm{y} \lambda_{12}$ & Término de varianza curvatura y diferenciación crucero (machos) & 0,01 \\
$\lambda_{13} \mathrm{y} \lambda_{14}$ & Término de varianza curvatura y diferenciación crucero (hembras) & 0,01 \\
$\lambda_{15}$ & Término de variación CV a la talla (machos) & $0,07 *$ \\
$\lambda_{16}$ & Término de variación CV a la talla (hembras) & $0,07 *$ \\
\hline
\end{tabular}

Tabla 3. Valores de los principales componentes de la función de máxima verosimilitud, función objetivo y Criterio de Información de Akaike (AIC).

Table 3. Value of the main log-likelihood functions in the overall objective function, and Akaike Information Criteria (AIC).

\begin{tabular}{|lrrrrrr|}
\hline Componente log-verosimilitud & modelo 1 & modelo 2 & modelo 3 & modelo 4 & modelo 5 & modelo 6 \\
\hline Función objetivo & 535,8 & 646,7 & 606,0 & 547,6 & 670,0 & 623,0 \\
Composición por talla pesquería machos & 116,7 & 175,3 & 161,3 & 122,1 & 171,6 & 158,6 \\
Composición por talla pesquería hembras & 154,6 & 170,3 & 146,8 & 163,5 & 185,3 & 161,8 \\
Composición por talla crucero machos & 73,7 & 107,3 & 109,9 & 73,0 & 112,0 & 113,2 \\
Composición por talla crucero machos & 75,5 & 97,8 & 95,9 & 75,8 & 97,8 & 95,4 \\
Número de parámetros & 112 & 112 & 112 & 192 & 192 & 192 \\
AIC & 759,8 & 870,7 & 830 & 931,6 & 1054 & 1007 \\
\hline
\end{tabular}


El modelo presentó un buen ajuste en machos y hembras prediciendo la estructura observada (Figs. 3 y 4). En machos, las mayores diferencias entre la composición observada y estimada se presentaron en 1992, 1993, 1994 y 1996, mientras que en hembras correspondieron a 1994, 1995, 1997 y 1999. Es probable que la flota arrastrera industrial intensifique la captura de ejemplares por sobre los $37 \mathrm{~cm}$ de longitud total y provoque que la fracción descartada sea poco representada. Sin embargo, el modelo de dinámica poblacional logra predecir la tendencia general en la estructura observada.

El modelo logra un adecuado ajuste a los datos, prediciendo la estructura de la población modelada (Figs. 5 y 6). En machos, los mayores problemas se presentaron en el ajuste del año 1993. Estos problemas se relacionaron básicamente con la presencia de dos modas (30 y $48 \mathrm{~cm}$ de longitud total), y con importantes irregularidades en la composición observada. En los otros años de la serie acústica, el ajuste no presentó mayores problemas prediciendo la estructura, con excepción de 2000 donde se observó claramente una composición bimodal, existiendo algunas diferencias entre la estructura observada y la predicha por el modelo.
En hembras, las mayores diferencias en el ajuste se presentaron en 1993 y 2000. En 1993 se observaron dos modas (32 y $55 \mathrm{~cm}$ de longitud total), mientras que el 2000 las modas fueron de 35 y 50 $\mathrm{cm}$. En los otros años de la serie las diferencias entre la composición por tamaños observada y estimada fueron menores, prediciendo la estructura.

El patrón de selectividad o patrón de explotación en los cruceros de evaluación acústica, presentó diferencias entre machos y hembras (Fig. 7). En machos el máximo se obtuvo a edades intermedias entre 6 y 8 años, mientras que en hembras el máximo se produjo a partir de la edad 9. Este patrón es coherente con las características de crecimiento de la merluza común, siendo las curvas de selectividad representativas de una pesquería de arrastre con un fuerte componente selectivo asociado a la edad del ejemplar y su tamaño, que lo hace más susceptible al arte de pesca.

La tasa de mortalidad por pesca de machos entre 1992 y 1994 fluctuó alrededor de 0,31 año-1. De 1994 a 1996, la tasa de mortalidad disminuyó para mantenerse en 1996 cercana a 0,12 año $^{-1}$, y se incrementó alcanzando a 0,17 año- ${ }^{-1}$ el 2000. En hembras el patrón fue similar, observándose la mayor
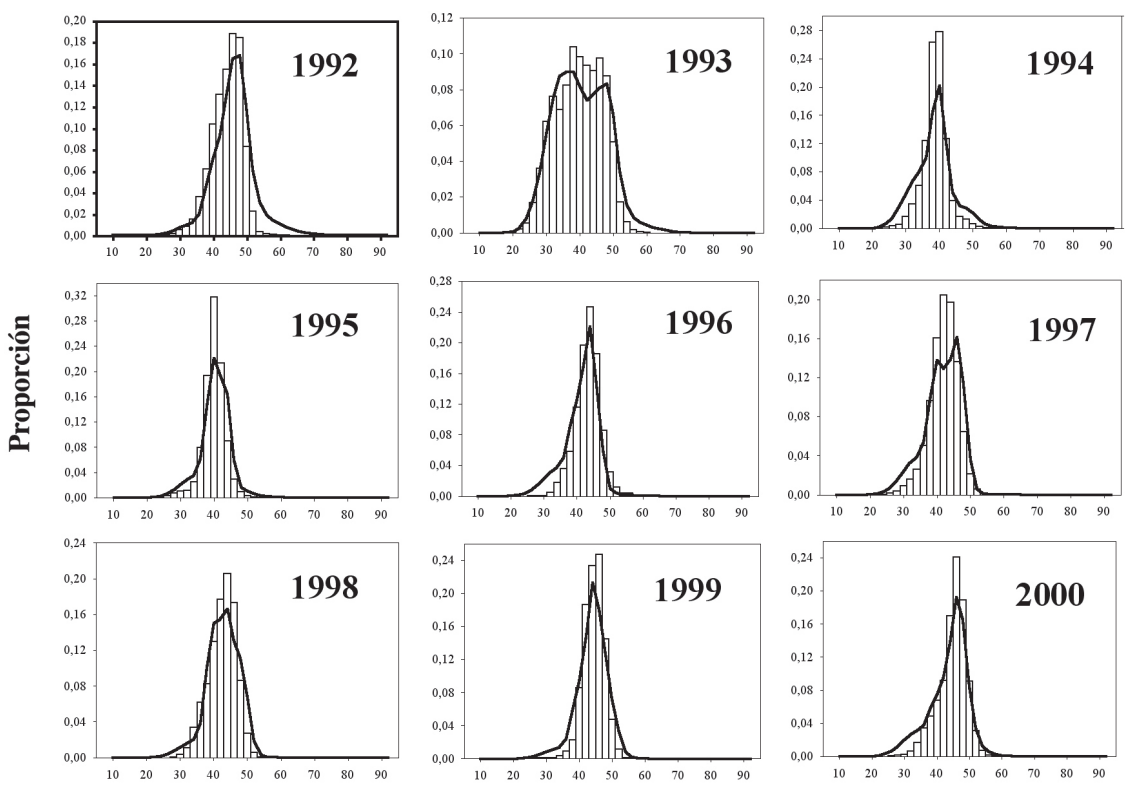

Intervalo clase $(\mathrm{cm})$

Figura 3. Composición de tallas de machos, observada (barras) y estimada (líneas), en la zona centro-sur de Chile.

Figure 3. Fishery size composition observed (bar) and estimated (line) for male of Chilean hake in the centralsouth of Chile. 

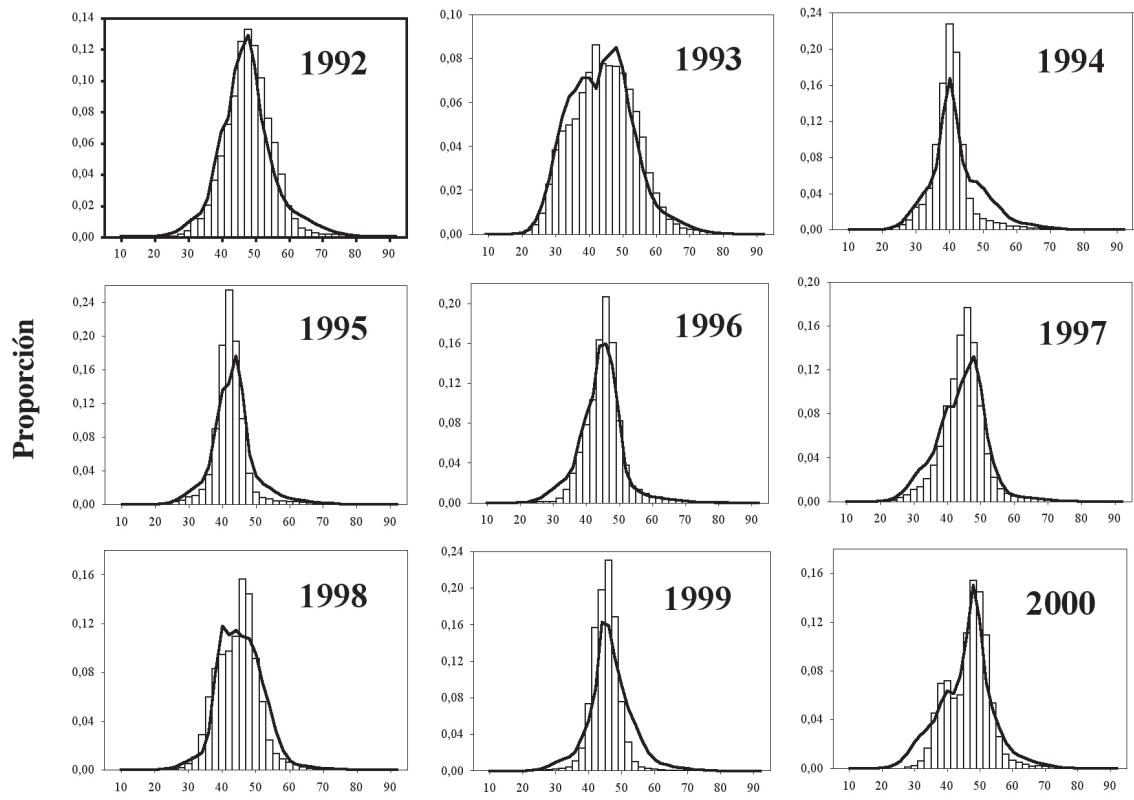

Intervalo clase $(\mathbf{c m})$

Figura 4. Composición de tallas en la pesquería de hembras, observada (barras) y estimada (líneas), en la zona centro-sur de Chile.

Figure 4. Fishery size composition observed (bar) and estimated (line) for females of Chilean hake in the central-south of Chile.

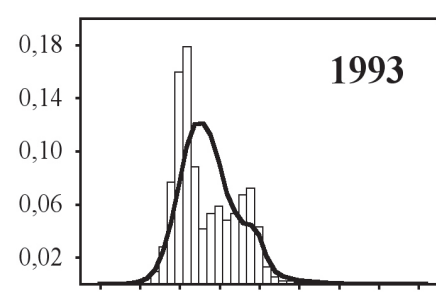

$102030 \quad 40 \quad 50 \quad 6070 \quad 80 \quad 90$
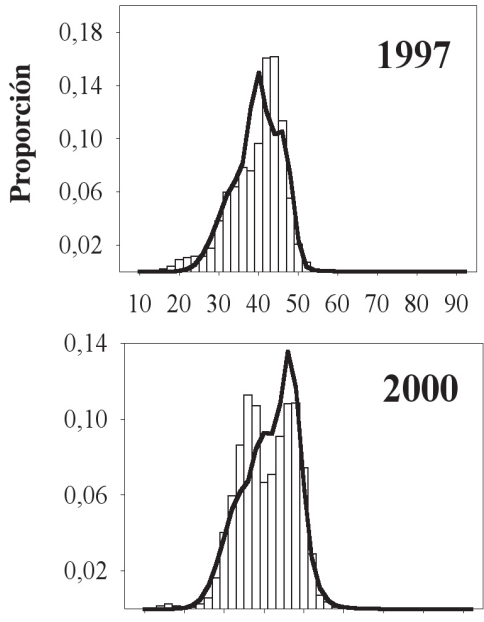

$10 \quad 2030 \quad 4050 \quad 6070 \quad 8090$

Intervalo clase $(\mathbf{c m})$
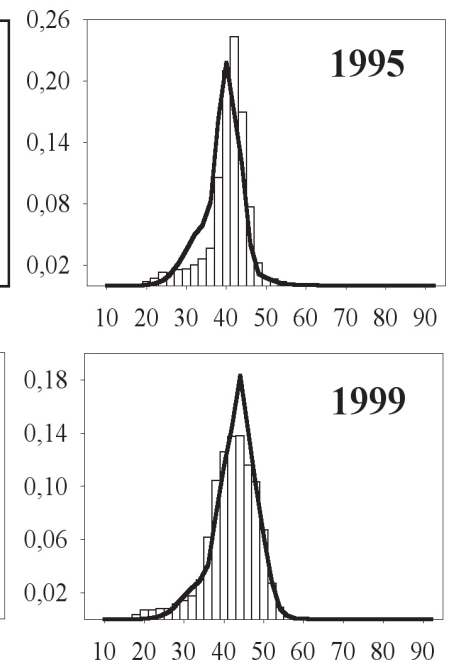

Figura 5. Composición de tallas de machos en los lances en los cruceros acústicos, valores observados (barras) y estimados (líneas), en la zona centro-sur de Chile.

Figure 5. Trawls survey size composition observed (bar) and estimated (line) for males of Chilean hake in the central-south of Chile. 


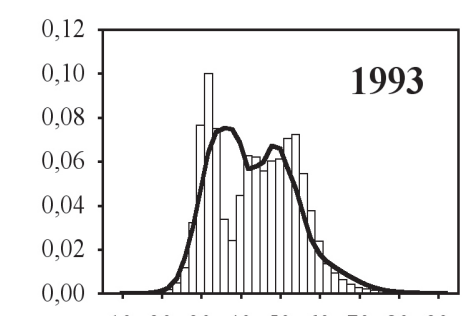

$\begin{array}{lllllllll}10 & 20 & 30 & 40 & 50 & 60 & 70 & 80 & 90\end{array}$

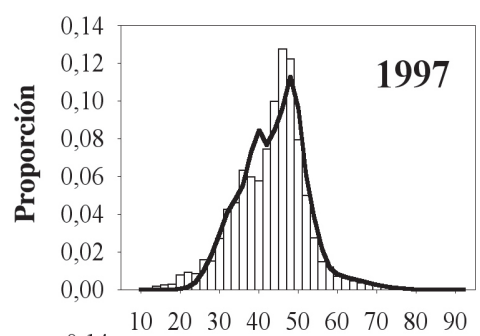

$\begin{array}{lllllllll}10 & 20 & 30 & 40 & 50 & 60 & 70 & 80 & 90\end{array}$

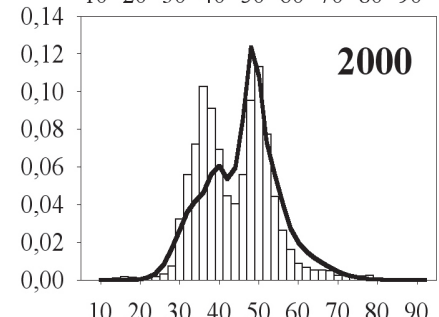

$\begin{array}{lllllllll}10 & 20 & 30 & 40 & 50 & 60 & 70 & 80 & 90\end{array}$

Intervalo clase $(\mathrm{cm})$

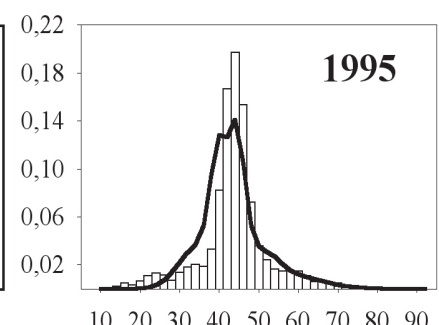

$\begin{array}{lllllllll}10 & 20 & 30 & 40 & 50 & 60 & 70 & 80 & 90\end{array}$

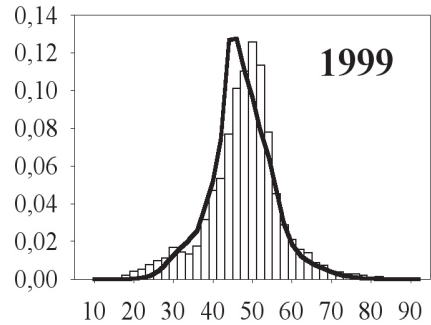

Figura 6. Composición de tallas de hembras en los lances en los cruceros acústicos, valores observados (barras) y estimados (líneas), en la zona centro-sur de Chile.

Figure 6. Trawls survey size composition observed (bar) and estimated (line) for females of Chilean hake in the central-south of Chile.

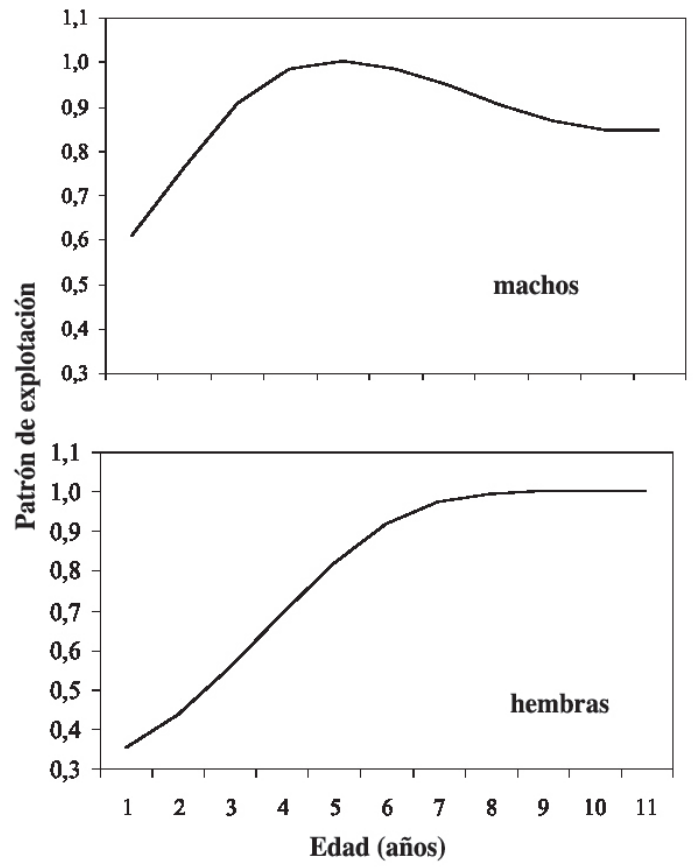

Figura 7. Patrón de selectividad en crucero acústico de machos y hembras.

Figure 7. Males and females selectivities for hydroacoustic surveys.

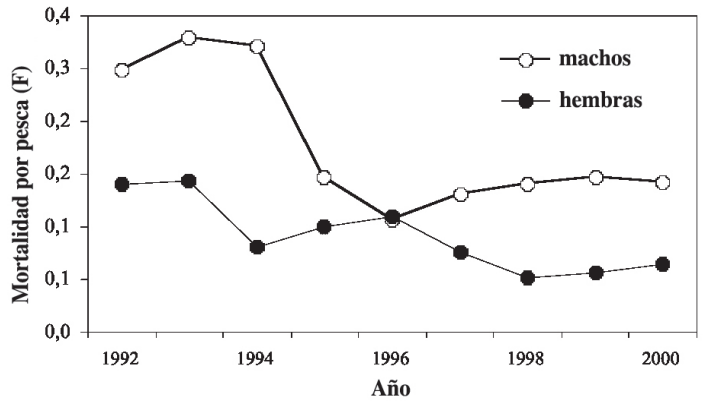

Figura 8. Tasas de mortalidad por pesca estimadas en merluza común entre 1992 y 2000.

Figure 8. Fishing Mortality rates estimated in Chilean hake between 1992 and 2000. 
mortalidad entre 1992 y 1993 con 0,16 año $^{-1}$ y 0,17 año-1 ${ }^{-1}$ espectivamente, bajando a niveles de 0,09 año${ }^{1}$ en 1994, donde se incrementó nuevamente llegando a 0,13 año $^{-1}$ en 1996. Posteriormente, la tasa de mortalidad por pesca disminuyó alrededor de 0,06 año ${ }^{-1}$ hacia el 2000 (Fig. 8).

Los estimados de abundancia de machos y hembras predicen de manera aceptable las tendencias observadas en los cruceros de prospección acústica. En machos la abundancia de ejemplares mostró una tendencia creciente entre 1993 y 1997, con un cambio en la tendencia al final de la serie bajando a niveles de abundancia cercanos a 500 millones de ejemplares. En hembras, la tendencia general fue positiva en toda la serie, finalizando en alrededor de 600 millones de individuos el 2000 (Fig. 9).

El patrón de reclutamiento estimado correspondiente a la abundancia del grupo de edad 3, mostró fuertes fluctuaciones en la magnitud de las distintas clases anuales que reclutan a la pesquería (Fig. 10). En el período de estudio, el nivel de reclutamiento más bajo se estimó en 1992 (123 millones de ejemplares). Luego, entre 1993 y 1996 se estimaron reclutamientos fuertes que fluctuaron entre 620 y 994 millones de reclutas. El mayor nivel de reclutamiento se estimó en 1997, con 1024 millones de ejemplares reclutados a la pesquería. En 1998 y 1999, los reclutamientos fueron moderados y finalizó la serie el 2000 con reclutamiento de 816 millones de reclutas.

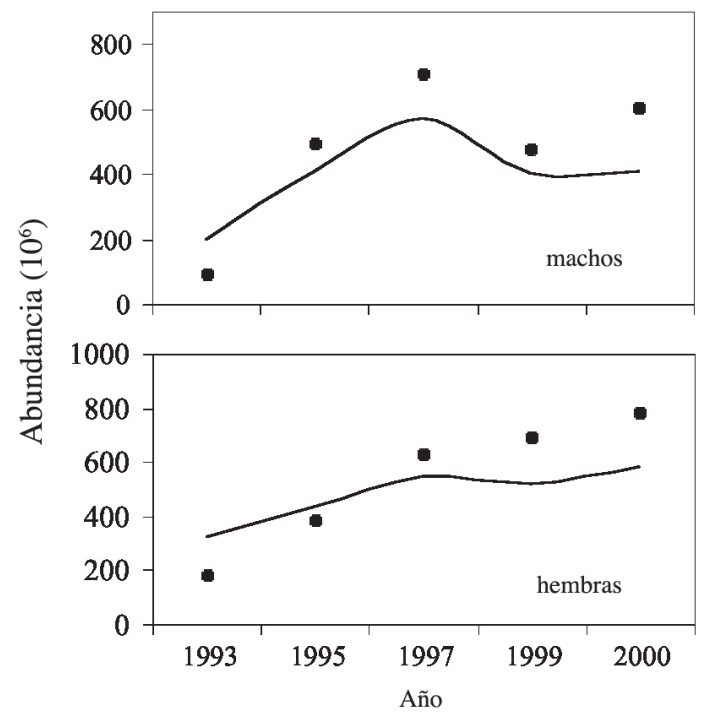

Figura 9. Abundancias observadas (punto) y estimadas (línea) en las evaluaciones acústicas.

Figure 9. Observed (point) and estimated (line) abundance for hydroacoustic evaluation.
La biomasa total en la serie modelada mostró un incrementó importante desde 1993 hasta 1997, para luego mantenerse alrededor de 1,3 millones de ton en el 2000. Con respecto a la biomasa desovante, cuantificada como la fracción de la población adulta que sobrevive al primero de agosto, se apreció un incremento considerable de sus niveles a partir de 1993, llegando a un máximo de 818 mil ton. Después, se observó un descenso el 2000 de alrededor de 785 mil ton (Fig. 11). La tendencia observada en

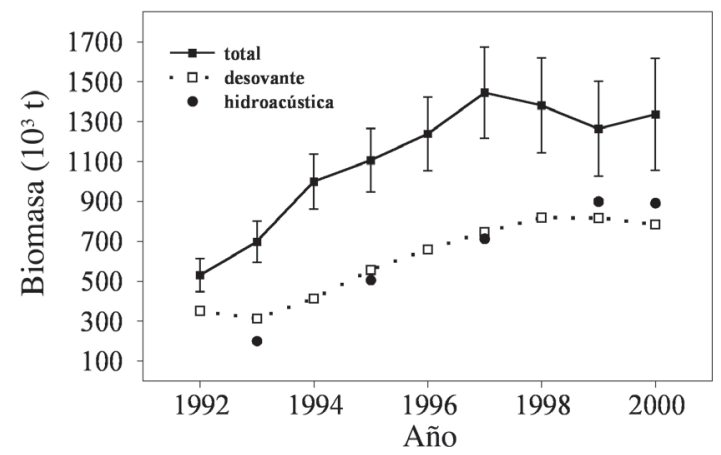

Figura 11. Cambios de biomasa total, biomasa desovante y biomasa acústica de merluza común entre 1992 y 2000. Se incluye el intervalo de confianza para el estimado anual de biomasa total (barra vertical).

Figure 11. Changes in total biomass, spawning biomass and acoustic biomass of Chilean hake from 1992 to 2000. The confidence interval es included in the annual estimate of total biomass (vertical bar).

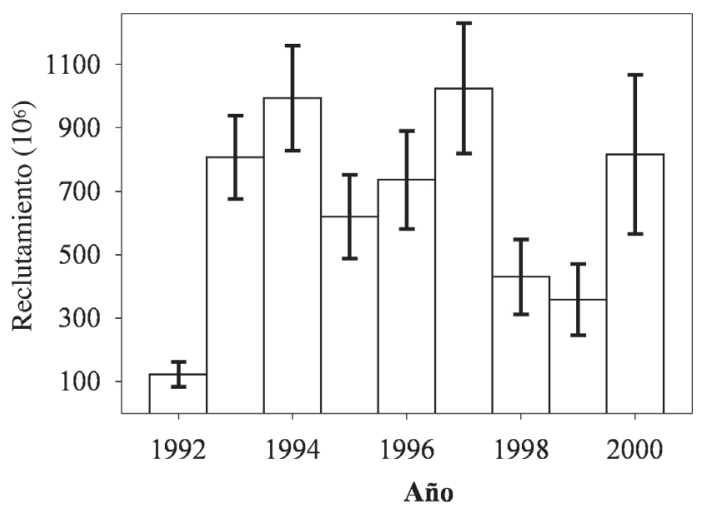

Figura 10. Abundancia en número del grupo de edad 3 de merluza común (reclutas) entre 1992 y 2000. Se incluye el intervalo de confianza para el estimado anual de reclutamiento (barra vertical).

Figure 10. Recruitment in number of age 3 between 1992 and 2000. The confidence interval is included in the estimate of annual recruitment (vertical bar). 
la biomasa desovante coincidió con las evaluaciones de los cruceros de prospección hidroacústica, indicando con ello una importante asociación entre estos indicadores de condición del stock.

\section{DISCUSIÓN}

La principal ventaja del enfoque de modelación implementado, está en la incorporación de la estructura poblacional subyacente en la composición por tallas de las capturas y de la población en los cruceros de evaluación hidroacústica. La calidad de los ajustes a la composición por tallas y la coherencia entre los distintos indicadores de condición del stock, indica que la información de frecuencia de tallas por sí sola es una pieza de información relevante sin que necesariamente deba ser convertida en composición por edad, previo a la evaluación del stock.

El modelo de evaluación no incorpora información auxiliar de datos de captura por unidad de esfuerzo (CPUE) en el modelo, debido a las fuertes variabilidades que se pueden presentar en el coeficiente de capturabilidad (Payá et al., 1992, 1993). Esta variabilidad hace que la relación entre la biomasa total y este índice no reflejen de manera adecuada las tendencias presentes en la naturaleza. Por otra parte, el sistema de cuotas de captura limita el uso de estos indicadores al no cumplirse los supuestos básicos asociados a la CPUE como índice de abundancia (Hilborn \& Walters, 1992; Quinn \& Deriso, 1999). Sobre este punto, el modelo hace la diferencia con las metodologías clásicas de evaluación, debido a que incorpora la composición por tamaños, bajo el supuesto de que existe una sola pesquería y que los patrones de crecimiento son un atributo de la población.

Debido a que los datos básicos relacionados con la dinámica poblacional se encuentran ajustados a la información de composición por tallas de la pesquería y de los cruceros de evaluación, el análisis de los cambios de abundancia es esencialmente estructurado por tamaño. En relación con el uso de la información acústica, se destaca que una forma sencilla de incorporación de estos datos es utilizar la biomasa total cuantificada, sin ser necesario el utilizar la biomasa transformada en abundancia mediante la estructura de tallas recolectada durante el crucero de evaluación. Sin embargo, se utilizó esta información para mantener el carácter diferencial en el crecimiento, mortalidad y vulnerabilidad de cada sexo. El modelo es bastante flexible para aco- modar varios tipos de información provenientes tanto de la pesquería como de los cruceros de evaluación acústica, lo que constituye una ventaja. Esta ventaja, se asocia básicamente con la existencia de un criterio estadístico en la estimación de los parámetros (Fournier \& Archibald, 1982; Deriso et al., 1985; Kimura, 1990), y aunque el proceso de estimación sea completamente objetivo, subyacen en el proceso algunos criterios que dependen de cada investigador. Estos criterios se relacionan con los factores de ponderación de los "lambdas" (términos de varianza), incorporados en la función de verosimilitud. En este sentido existen varias alternativas de manejo de la información y de la ponderación asignada a cada componente de verosimilitud, siendo el criterio básico el grado de incertidumbre que se tenga con cada pieza de información. En definitiva es un proceso de búsqueda para conseguir que las observaciones sean bien representadas por el modelo.

Los resultados del análisis talla-estructurado sugieren que la población de merluza común se encuentra en un período de alta abundancia, producto de clases anuales fuertes en 1993, 1994, 1997 y 2000, y mortalidades por pesca bajas a moderadas entre 1997 y 2000. Esta situación del estado de la población está en acuerdo con los resultados de otras evaluaciones indirectas y análisis de riesgo (Gálvez et al., 1996; Payá et al., 1998). Este escenario habría favorecido un incremento de la biomasa total, adulta y desovante, indicando además que la variabilidad en la biomasa total está fuertemente influenciada por la fuerza de las clases anuales que reclutan a la pesquería. En este sentido, es altamente recomendable mantener una constante actualización de los indicadores de condición del stock, y en especial de los cambios de reclutamiento y de biomasa desovante, con el objeto de conocer la condición del stock. Uno de los resultados interesantes radica en la fuerte asociación entre los cambios y tendencias en la biomasa desovante y la biomasa cuantificada acústicamente. En efecto, la evaluación con métodos acústicos debe revelar la biomasa total presente; no obstante, la salida del modelo sugiere que más bien es la biomasa desovante la que está siendo evaluada en agosto de cada año. Esta situación es consecuencia directa de suponer que $q^{H}=1$, sin embargo no se exploraron hipótesis alternativas tal que permitan señalar qué fracción de la biomasa total de merluza común está representando las evaluaciones acústicas, aspecto que deberá ser abordado en futuros estudios. 
Desde el punto de vista de los autores, la principal desventaja que tiene el modelo de evaluación utilizado se encuentra en considerar, al igual que en la mayoría de los modelos de evaluación de stock, que la tasa de mortalidad natural es constante para todas las clases de edad (Schnute, 1987; Megrey \& Wespestad, 1988), aun cuando se han demostrado importantes sesgos en las estimaciones como producto de este supuesto en poblaciones edadestructuradas (Sims, 1984; Mertz \& Myers, 1997). En este contexto, la merluza común se caracteriza por ser el principal predador del sistema demersal de Chile central (Meléndez, 1984; Arancibia, 1989; Fuentealba, 1993). Esta especie presenta alto canibalismo (Fuentealba, 1993), el cual puede estar relacionado con la intensidad de las clases anuales que reclutan al stock explotable (Arancibia et al., 1998). Esta característica es común en este tipo de especies: M. productus (Livingston et al., 1986), $M$. capensis y $M$. paradoxus del sur de Africa (Macpherson \& Roel, 1987; Punt et al., 1992), y Merluccius gayi peruanus (Espino \& WosnitzaMendo, 1989; Castillo et al., 1989). En Chile el trabajo de Arancibia et al. (1998) indica que los modelos de evaluación para el stock de merluza común en Chile centro-sur (Paya et al., 1992, 1993; Cubillos et al., 1994), presentan una alta incertidumbre asociada a la cuantificación de los reclutamientos de merluza común. Es necesario destacar que el canibalismo no es la única fuente de mortalidad en juveniles. No obstante, existe evidencia que indica que el canibalismo podría estar relacionado con la intensidad de las clases anuales (Livingston \& Lang, 1996), constituyéndose en un importante mecanismo de regulación poblacional (Laevastu \& Favorite, 1988), que podría estar actuando en el caso de la merluza común.

Otra desventaja del enfoque de análisis es la carencia de variabilidad climática incorporada al análisis y modelación de los procesos. En el caso que una hipótesis de mayor intensidad en el canibalismo esté asociada con una mayor sobreposición entre las fracciones de las poblaciones adultas y juveniles sea correcta, saber qué condiciones ambientales favorecerían dicho proceso, sería un aporte al conocimiento de la dinámica poblacional del recurso. Wespestad et al. (2000), estudiando el walleye pollock (Theragra chalcogramma) encontraron una relación entre canibalismo, variabilidad climática, transporte físico y reclutamiento en el mar de Bering. Por lo tanto, hay razones para suponer que el ambiente desempeña un papel importante al generar las condiciones bajo las cuales se produce canibalismo en la merluza común.

\section{AGRADECIMIENTOS}

El primer autor agradece las facilidades otorgadas por el Instituto de Investigación Pesquera (INPESCA) para desarrollar esta evaluación relacionada con el trabajo de Tesis de Postgrado presentado en el programa de Magíster en Ciencias Mención en Pesquerías de la Universidad de Concepción. Los autores agradecen las sugerencias y comentarios de los revisores anónimos del manuscrito, que ayudaron a mejorar la presentación y exposición metodológica de esta evaluación de stock para la merluza común.

\section{REFERENCIAS}

Aguayo, M. \& V. Ojeda. 1987. Estudios de la edad y crecimiento de merluza común (Merluccius gayi Guichenot, 1848) (Gadiformes-Merlucciidae). Invest. Pesq. (Chile), 34: 99-112.

Arancibia, H. 1989. Distribution patterns, trophic relationships and stock interactions in the demersal fish assemblage off central Chile. Dr. Rer. Nat. Thesis, University of Bremen, RFA, 211 pp.

Arancibia, H., M. Catrilao \& B. Farías. 1998. Evaluación de la demanda de alimento en merluza común y análisis de su impacto en pre-reclutas. Informe Final Proyecto FIP 95-17: 98 pp.

Beyer, J. 1991. On the length-weight relationship. Part II. Computing mean weights from length statistics. Fyshbite, 9(2): 50-54.

Castillo, R., L. Juárez \& L. Higgison. 1989. Predación y canibalismo en la población de la merluza peruana de la zona de Paita. En: R. Jordán, R. Kelly, O. Mora, A.Ch. de Vildoso \& N. Henriquez (eds.). Memorias del Simposio Internacional de los Recursos Vivos y Pesquerías del Pacífico Sudeste, Rev. Pacífico Sur (Número especial), 273-286.

Cerna, J.F. \& C. Oyarzún. 1998. Talla de primera madurez sexual y fecundidad parcial de la merluza común (Merluccius gayi, Guichenot 1848) del área de la pesquería industrial de la zona de Talcahuano, Chile. Invest. Mar., Valparaíso, 26: 31 40. 
Cubillos, L., P. Sobarzo \& H. Arancibia. 1994. Análisis retrospectivo de la evaluación de merluza común (Merluccius gayi) de Chile centro-sur utilizando análisis secuencial de la población sintonizado. Doc. Téc. Inst. Invest. Pesq. (IIP), Talcahuano, 3(5): 1-24.

Deriso, R.B., T.J. Quinn II \& P.R. Neal. 1985. Catchage analysis with auxiliary information. Can. J. Fish. Aquat. Sci., 42: 815-824.

Doubleday, W.G. 1976. A least squares approach to analyzing catch at age data. Int. Comm. Northw. Atl. Fish. Res. Bull., 12: 69-81.

Espino, M. \& V. Wosnitza-Mendo. 1989. Relación entre las poblaciones de merluza (Merluccius gayi peruanus) y anchoveta (Engraulis ringens). En: R. Jordán, R. Kelly, O. Mora, A.Ch. de Vildoso \& N. Henríquez (eds.). Memorias del Simposio Internacional de los Recursos Vivos y Pesquerías del Pacífico Sudeste. Rev. Pacífico Sur (Número especial), 267-271.

Fournier, D. \& C.P.Archibald. 1982. A general theory for analyzing catch at age data. Can. J. Fish. Aquat. Sci., 39: 1195-1207.

Fournier, D.A., J.R. Sibert, J. Majkowski \& J. Hampton. 1990. MULTIFAN a likelihood-based method for estimating growth parameters and age composition from multiple length frequency data sets illustrated using data for bluefin tuna (Thunnus maccoyii). Can. J. Fish. Aquat. Sci., 47: 301-317.

Fréon, P. \& O.A. Misund. 1999. Dynamics of pelagic fish distribution and behaviour: effects on fisheries and stock assessment. Fishing News Book, New York, $348 \mathrm{pp}$.

Fuentealba, M. 1993. Relaciones tróficas intraespecíficas de la merluza común, Merluccius gayi (Guichenot, 1948) de Chile central. Tesis de Magíster en Ciencias con Mención en Zoología, Universidad de Concepción, Chile, 110 pp.

Gálvez, M., L. Cubillos \& R. Alarcón. 1996. Análisis de la pesquería de merluza común (Merluccius gayi), evaluación del stock y estimación de la captura total permisible para 1997. Doc. Téc, Inst. Invest. Pesq. (IIP), Talcahuano, 5(1): 1-43.

Hilborn, R. \& C.J. Walters. 1992. Quantitative fisheries stock assessment, choice, dynamics and uncertainty. Chapman \& Hall, New York, 570 pp.

Hilborn, R. \& M. Mangel. 1997. The ecological de- tective confronting models with data. Princeton University Press, New Jersey, 315 pp.

Ianelli, J. \& M. Zimmerman. 1998. Status and future prospects for the Pacific Ocean perch resource in waters off Washington and Oregon as assessed in 1998. AFSC, NMFS, NOAA, Seattle, Washington, $54 \mathrm{pp}$.

Kimura, D.K. 1990. Approaches to age-structured separable sequential population analysis. Can. J. Fish. Aquat. Sci., 47(12): 2364-2374.

Laevastu, T. \& F. Favorite. 1988. Fishing and stock fluctuation. Fishing Books, Farnham, 239 pp.

Lillo, S., J. Giakoni., A. Paillamán, I. Payá, S. Mora, C. Cerda, J. Blanco \& H. Arancibia. 1993. Evaluación directa del stock de merluza común en la zona centro-sur. Informes Técnicos FIP. FIP-IT/9303: $131 \mathrm{pp}$.

Lillo, S., J. Córdova, M. Rojas, V. Ojeda, J. Olivares, R. Tascheri, R. Alarcón, L. Miranda, M. Braun, S. Núñez \& J. Ortiz. 1998. Evaluación hidroacústica del stock de merluza común explotado en la zona centro-sur. Informes Técnicos FIP. FIP-IT/97-12: 125 pp.

Lillo, S., R. Vega, V. Ojeda, J. Olivares, R. Tascheri, M. Braun, V. Valenzuela, S. Núñez \& J. Ortiz. 2000. Evaluación acústica del recurso merluza común en la zona centro-sur. Informes Técnicos FIP. FIP-IT/99-04: 132 pp.

Lillo, S., S. Núñez, V. Ojeda, F. Balbontín, M. Braun, R. Tascheri, A. Saavedra, R. Bravo, J. Ortiz, P. Torres, L. Cubillos \& J. Olivares 2001. Evaluación hidroacústica de merluza común, 2001. IFOP. Informe Final. FIP N²001-18: 166 pp.

Livingston, P.A., D.A. Dwyer, D.L. Wencker, M.S. Yang \& G.M. Lang. 1986. Trophic interactions of the key fish species in the Eastern Bering Sea. Inst. North Pacific Fish Comm. Bull., 47: 49-65.

Livingston, P.A. \& G.M. Lang. 1996. Interdecadal comparisons of walleye pollock, Theragra chalcogramma, cannibalism in the eastern Bering Sea. In: U.S. Dep. Commer. NOAA Tech. Rep. NMFS, 126: 115-124.

Macpherson, E. \& B. Roel. 1987. Trophic relationships in the demersal fish community of Namibia. En: A.I. Payne, J.A. Gulland \& K.H. Brink (eds.). The Benguela and comparable ecosystems. S. Afr. J. Mar. Sci., 5: 585-596. 
Megrey, B.A. 1989. Review and comparisons of agestructured stock assessment models from theoretical and applied points of view. American Fisheries Society Symposium, 6: 8-48.

Megrey, B. \& V. Wespestad. 1988. A review of biological assumption underlying fishery assessment models. En: W.S. Wooster (ed.). Fishery science and manegament: objetives and limitations. Lectures notes on coastal and stuarines studies 28. Springer-Verlag, pp. 31-69.

Meléndez, R. 1984. Alimentación de Merluccius gayi (Guichenot) frente a Chile central $\left(32^{\circ} 05^{\prime} \mathrm{S}\right.$ $36^{\circ} 50^{\prime}$ S). Bol. Mus. Nac. Hist. Nat., Chile, 54 pp.

Mertz, G. \& R. Myers. 1997. Influence of errors in natural mortality estimates in cohort analysis. Can. J. Fish. Aquat. Sci., 54: 1608-1612.

Methot, R.D. 1986. Synthetic estimates of historical abundance and mortality for northern anchovy, Engraulis mordax. U.S. Natl. Mar. Fish. Serv., Southwest Fish. Cent., Admin. Rep. LJ 86-29, La Jolla, California.

Ojeda, V., J. Olivares, F. Balbontín, D. Garland, L. Cubillos, R. Alarcón, M. George-Nascimento \& A. Sepúlveda. 1997. Validación de los métodos aplicados en la estimación de edad y crecimiento, y determinación de la mortalidad en merluza común en la zona centro-sur. Informe Final, FIP 9515: $129 \mathrm{pp}$.

Payá, I., J. Sateler, J. Donoso \& S. Mora. 1992. Diagnóstico de las principales pesquerías nacionales 1991. Pesquerías demersales, peces. Zona centrosur. Estado de situación y perspectivas del recurso (SGI-IFOP 92/3). Corporación de Fomento de la Producción. Instituto de Fomento Pesquero, 52 pp.

Payá, I., J. Sateler, J. Donoso \& S. Mora. 1993. Diagnóstico de las principales pesquerías nacionales 1992. Pesquerías demersales, peces. Zona centrosur. Estado de situación y perspectivas del recurso (SGI-IFOP 93/2). Corporación de Fomento de la Producción. Instituto de Fomento Pesquero, 41 pp.
Payá, I., S. Lillo, A. Paillamán, S. Mora, V. Ojeda, E. Figueroa, J. Blanco, H. Arancibia, R. Alarcón, L. Miranda, A. Pacheco \& L. Cubillos. 1995. Evaluación hidroacústica del stock de merluza común en la zona centro-sur. Informes Técnicos FIP. FIP IT/95-14: 73 pp.

Payá, I., O. Oyarzún, R. Galleguillos \& M. Oliva. 1996. Unidades de stock en el recurso merluza común de la zona centro-sur. Pre-Informe final, FIP 94-18: 83 pp.

Payá, I., N. Ehrhardt \& M. Aguayo. 1998. Estrategia de explotación de merluza común en la zona centro-sur, bajo incertidumbre del tamaño y rendimiento sustentable del stock. Pre-Informe final, FIP 97-11: $101 \mathrm{pp}$.

Punt, A., R. Leslie \& S. Du Plessis. 1992. Estimation of the annual comsumption of the food by Cape hake Merluccius capensis and M. paradoxus off the South African west coast. Benguela Trophic Functioning. S. Afr. J. Mar. Sci., 12: 611-634.

Quinn II, T.J. \& R.B. Deriso. 1999. Quantitative fish dynamics. Oxford University Press, New York, 542 pp.

Schnute, J. 1987. A general fishery model for a sizestructured fish population. Can. J. Fish. Aquat. Sci., 44: 924-940.

Schnute, J.T. \& L. Richards. 1995. The influence of error on population estimates from catch-age models. Can. J. Fish. Aquat. Sci., 52: 2063-2077.

Sims, S.E. 1984. An analysis of the effect of errors in the natural mortality rate on stock-size estimates using virtual population analysis (cohort analysis). J. Cons. Perm. Int. Explor. Mer, 41: 149-153.

Wespestad, V., L. Fritz, W. Ingraham \& B. Megrey. 2000. On relationships between cannibalism, climate variability, physical transport, and recruitment succes of Bering Sea walleye pollock (Theragra chalcogramma). ICES. J. Mar. Sci., 57: 268-274. 
Investigaciones Marinas, Vol. 32(1) 2004 